\title{
Correlation Study between Skeletal Scintigraphy and CT Scan in Diagnosing Bone Metastases
}

\author{
Saeed Bafaraj, Ali Algamdi, Abdulaziz Almorjan \\ Department of Diagnostic Radiology, Faculty of Applied Medical Science, King Abdulaziz University, Jeddah, \\ KSA \\ Email: smbafaraj@kau.edu.sa
}

Received 27 September 2014; revised 24 October 2014; accepted 24 November 2014

Copyright (C) 2014 by authors and Scientific Research Publishing Inc.

This work is licensed under the Creative Commons Attribution International License (CC BY).

http://creativecommons.org/licenses/by/4.0/

(c) (i) Open Access

\begin{abstract}
A retrospective study for 98 patient suspected to bone scan at department of radiology take place, to find out which imaging modality is more sensitive between computed tomography (CT) and bone scan; age group of patient was from 1 year to 90 year, the highest Site of Metastases for both Bone scan \& CT was at vertebra with $31 \%$, and the positive finding was also $31 \%$ for bone scan, while for CT the highest Site of Metastases was at forearm with 34\%, and the sensitivity for CT was $44 \%$, and $56 \%$ for bone scan. The study recommends that patients with bone metastases are kindly recommended to bone scan investigation rather than CT scan.
\end{abstract}

\section{Keywords}

\section{Skeletal Scintigraphy, CT Scan, Bone Metastases}

\section{Introduction}

Bone scanning was established in the late 1960s, and the introduction of 99mTc labelled bone seeking radiopharmaceuticals assist in saving time and effort, in addition to improved image quality. The clinical value of dynamic and static bone scanning are accepting among clinicians.

"Bone metastases can be a difficult concept to grasp”, says Julie Fasano, MD, a medical oncologist with Memorial Sloan-Kettering Cancer Center's Commack Facility in Long Island, NY. Although it usually shows up within two to three years of diagnosis, it can appear many years later, she says. Sometimes, it doesn't cause any symptoms.

Metastasis can occur when cancer cells break away from the primary tumor, where the cancer began. The cells may then enter the bloodstream or lymph system and travel to the bone marrow. "The matrix of the bone

How to cite this paper: Bafaraj, S., Algamdi, A. and Almorjan, A. (2014) Correlation Study between Skeletal Scintigraphy and CT Scan in Diagnosing Bone Metastases. Open Journal of Medical Imaging, 4, 178-186.

http://dx.doi.org/10.4236/ojmi.2014.44025 
marrow secretes cytokines”, Fasano says. These proteins may attract cancer cells.

Cancer cells can remain hidden and inactive in bone for a long time. This means they can evade treatment. At some point, however, the cells may begin to multiply and grow new blood vessels to obtain oxygen and food. This allows a tumor or tumors to form.

Scientists are just beginning to understand what happens in the bone to prompt this process, Fasano says. Once metastasis begins, there may be a "vicious, self-perpetuating cycle". The release of cytokines may attract yet more cancer cells to the bone marrow, and this may help cancer cells survive (Annie Stuart WebMD Feature Reviewed by Louise Chang, MD).

\section{Background}

The nuclear medicine is a type of imaging modalities but, differs from other modalities, where the patient is the source of radiation after we administrate radionuclides to him. It uses special type of equipment called gamma camera. Inside the gamma camera there is scintillation detector which counts and absorbs the photons from the patient then converts them to visible light.

\section{Research Plan}

\subsection{Objectives}

To find out which radiology modalities is more sensitive in diagnosing bone metastases.

\subsection{Material and Methods}

Data records and information regarding bone scan and CT at Department of nuclear medicine, were collected.

\subsection{Study Area}

Nuclear Medicine Department at Hospitals.

\subsection{Study Design}

Retrospective study to analyze the data of bone scan records done at nuclear medicine departments in correlation with CT scan.

\subsection{Sample Size}

Up to 100 patients records will be analyzed in this study.

\subsection{Data Collection Methods}

Electronically and hard copy data record of bone scan and CT scan results including patients details, protocols, indications, diagnosis, finding, ...etc. were collected.

\subsection{Data Management and Analysis Plan}

Using SPSS statistical program version 19, data analyzed.

\section{Skeletal System}

The skeletal system also called the skeleton weights only about 20 pounds (9 kilograms) in human adults, but is made up of 206 bones. Bones differ in size, shape, weight, and in some cases, what they are made of. This variety is due to the different functions of the skeleton: to provide the framework and support of the body, protect the tissues and organs in the body cavities, act as levers for muscle action, and provide a site for blood cell production [1].

\subsection{Anatomy}

The adult skeleton consists of 206 bones that hold you upright and protect your vital organs [1] and the skeleton 
of a newborn baby may contain as many as 300 bones, but many fuse (join together) during childhood [2]. Skeleton is divided in to tow main groups of bones- the axial skeleton and the appendicular skeleton [1].

\subsection{The Axial Skeleton}

The first structure of the axial skeleton is the skull, which is the bony framework of the head. The skull houses the brain and many sensory organs. The tow main areas of the skull are the cranium and the face. The skull contains twenty-two bones, many which are paired. The face contains fourteen bones. The lower jaw bone called the mandible is the skull's only moveable bone and the only one not directly connected to the other bones of the skull. In an adult, the skull and the upper body are supported by the twenty-six bones of the vertebral column. (each of the twenty-six bones is called a vertebra) the vertebral column-or spine-runs along the back of the body and connects to the bones of the chest, or thorax. In frontal, the thorax has a three part bone called the sternum — or breastbone-and twelve pairs of ribs. The ribs connect the sternum to the vertebral column [1].

\subsection{The Appendicular Skeleton}

The upper portion of the appendicular skeleton is composed of the pectoral or shoulder, girdle and the arm bones. The pectoral girdle consists of two bones-the collarbone, also called the clavicle, in the front, and a flat, triangular bone called the scapular, or shoulder blade, at the back of the body.

Articulating — or connecting at a joint—with the pectoral girdle is the upper arm bone called the humerus. The two lower arm bones, the radius and the ulna, connect with the humerus at the elbow. The wrist contains eight small carpal bones arranged in two rows of four. Five metacarpal bones form the framework for the palm of each hand.

Each finger bone is called a phalanx. There are fourteen phalanges (plural for phalanx) in each hand-two for the thumb and three for each finger.

The lower portion of the appendicular skeleton is composed of the pelvic girdle and the leg bones. The pelvis, or hip bones appears to be just one bone, but it is actually composed of three bones called the ilium, ischium and pubis. The lower extremities - the leg-connect to the pelvic girdle at the acetabulum socket. Each leg is formed by the femur (thigh bone) the patella (kneecap) and the two lower leg bone, the tibia (shin bone) and the fibula.the structure of foot is similar to the hand, but it is much stronger. The ankle contains seven tarsal bones and the foot has five metatarsals. Like finger, the toed contain fourteen phalanges [1].

\subsection{What the Bone Made of?}

Bones are not empty and lifeless - they are dynamic, living organs. although the spaces between the living cells of bone tissue are filled with organic salts—such as calcium carbonate (7 percent) and calcium phosphate (85 percent) and small amount of sodium and magnesium [1].

\subsection{Physiology}

The skeletal system serves as an important mineral reserve. For example, if blood levels of calcium or magnesium are low and the minerals are not available in the diet, they will be taken from the bones. On the other hand the skeletal system provides Calcium needed for all muscle contractions. Lymphocytes and other cells relating to the immune response are produced and stored in the bone marrow. The skeletal system aids in protection of the nervous system, endocrine organs, chest and pelvic regions-all of these are vital organs [3]. At the center of some bones there is spongy tissue called red bone marrow that produces all the red blood cells and some of the white blood cells in the body. Adult bones also contain yellow bone marrow tissue which is mainly made up of fat cells [2].

\subsection{Pathology}

\subsubsection{Paget's Disease}

Paget's disease is a common disorder of bone architecture, of unknown aetiology, which occurs with increasing frequency after middle age. It is characterized initially by bone resorption, followed by a reparative process in which increased bone deposition results in bone expansion and abnormal modeling [4]. 


\subsubsection{Bone Tumor}

Benign bone tumors are generally well defined and have a narrow zone of transition between normal and abnormal bone. They cause signs and symptoms by expansion and pressure on adjacent structures. Primary malignant bone tumors are uncommon: they are destructive, often associated with periosteal reactions, and have a wide zone of transition between normal and abnormal bone [4].

\subsubsection{Bone Metastases}

Bone metastases are the most common malignant bone tumors. Metastases disseminate mainly to marrow-containing bones, therefore they are more commonly found in the axial skeleton. Any primary tumor may metastasize to bone, but the most frequent to do so are breast, prostate, lung, kidney and thyroid [4].

\subsubsection{Multiple Myeloma}

Multiple myeloma is a primary malignant tumor of bone marrow, in which there is infiltration of the marrow-producing areas of the skeleton by a malignant proliferation of plasma cells [4].

\subsubsection{Osteoporosis}

Osteoporosis is a condition in which there is a reduction of bone mass [4].

\subsubsection{Osteoarthritis}

Osteoarthritis is characterized by degeneration of articular cartilage and is part of the normal ageing process due to wear and tear of the articular surface [4].

\subsubsection{Rickets}

Vitamin D deficiency in children can cause rickets. Deficiency may be nutritional, due to malabsorption, chronic renal disease or prolonged anticonvulsant therapy [4].

\subsubsection{Osteomyelitis}

Osteomyelitis is an infection of bone, staphylococcus aureus being responsible for the majority of cases; other causes include tuberculosis and salmonella infection in sickle cell disease [4].

\subsubsection{Fractures}

Any bone may broken but some are particularly susceptible [4].

\subsubsection{Spondylolisthesis}

Spondylolisthesis refers to a slip of one vertebra on another, usually forwards but it may occasionally be backwards [4].

\section{Radiologic Examination}

\subsection{Role of Nuclear Medicine}

Nuclear medicine plays a crucial role in the diagnosis and management of various skeletal diseases because of the ability of scintigraphy to reflect changes in bone physiology. This permits the early identification of diseases and injuries. The increasing use of this imaging modality for the investigation of benign bone disorders is noteworthy. Utilization and effective use of these modalities should be based on a basic understanding of bone anatomy and physiology, technical aspects of nu-clear medicine techniques and sources of errors in con-ducting and interpreting these modalities [5].

\subsubsection{Bone Scan}

Strontium-85, strontium-87m and fluorine-18 were used as bone imaging radiopharmaceuticals before the introduction of technetium-99m to medicine in 1964. The first Tc99m-labeled bone imaging agent to be described was stannous-reduced Tc99m tripolyphosphate, followed by several other compounds with a higher bone uptake. The first was Tc99m pyrophosphate, shows many patterns in a variety of benign and malignant bone diseases 
(some may be specific). Many of these patterns are better understood once the underlying pathophysiological changes are appreciated and the clinical condition of the patient is known. Paying attention to the clinical and technical details can make the imaging results more specific than just localizing an abnormality [5].

\subsubsection{Protocol Used in Nuclear Medicine Department}

- Radiopharmaceutical used ${ }^{99 \mathrm{~m}} \mathrm{Tc}-\mathrm{MDP}$ (methylene diphosphonate) and ${ }^{99 \mathrm{~m}} \mathrm{Tc}-\mathrm{HDP}$ (hydroxymethylene diphosphonate),

- Dose: 20 mci

- Method of administration: intravenously

- 3-phase bone scan

- Patient Drink 2 glasses of fluid before attending the procedure

- Position: Supine

- Collimator: LEAP Energy 120/20 window

- Imaging:

- Sequential images: 4 secs/frame for 20 frames NOTE: collimator will be placed at the ROI.

- Blood Pool image: 1000 Kcts NOTE: patient will be instructed to drink 1 glass of water every 30 mins (6 glasses) and empty the bladder.

- Delayed images 2 - 3 hours PIT.

- Wholebody (ant. And post.)

- Static images (ant. And post.) 1000 Kcts

o chest, abdomen and pelvis

- static images $500 \mathrm{Kcts}$

o both hands, knees, arms and feet

o skull (anterior and lateral)

- NOTE: additional static view will be taken if needed

- Tomogram if indicated

- X-ray if needed

- Delayed static images 24 hours PIT

\subsubsection{Bone Scintigraphy for Metastases}

- Patient preparation: Drink 2 glasses of fluid before attending the procedure

- Position: Supine

- Collimator: LEAP Energy 120/20 window

- radioagent: 20 Mci Tc-99m MDP IV.

- imaging:

o Sequential images:

- Option 1: Sequential images: 4 secs/frame for 20 frames, NOTE: collimator will be placed at the ROI.

- Option 2, .No need for dynamic, Blood Pool image: 1000 Kcts, NOTE: patient will be instructed to drink 1 glass of water every 30 mins (6 glasses) and empty the bladder.

o Delayed images 2 - 3 hours PIT.

- Wholebody (ant. And post.)

- Static images (ant. And post.) $1000 \mathrm{Kcts}$, *chest, abdomen and pelvis

- Static images 500 Kcts, both hands, knees, arms and feet, skull (anterior and lateral), NOTE: additional Static view will be taken if needed

- Tomogram if indicated

- X-ray if needed

\subsubsection{Skeletal System Red Marrow Scintigraphy}

- Whole-body: (Anterior and Posterior)

- Static images: 300 - 500 Kcts

o Skull (anterior)

o Chest (anterior and posterior) 
o Abdomen (anterior and posterior)

o Both arms (anterior)

o Pelvis (anterior and posterior)

o Both femurs (anterior)

o Both knees (anterior)

o Both tibia (anterior)

o Both feet (anterior)

- Tomogram if indicated

\subsection{CT}

Application of computed tomography (CT) for examination of the skeletal system has developed slowly. This lag has occurred primarily because CT was not expected to be useful in scanning the skeletal system. However, the difference between the density of bone and that of soft tissue and the absence of motion artifacts make this system well suited for CT examination. It has proved useful in the evaluation of patients with primary bone neoplasm and those with skeletal metastases figure [6].

\section{Data Analysis and Results (Figures 1-7)}

\section{Bone Scan}

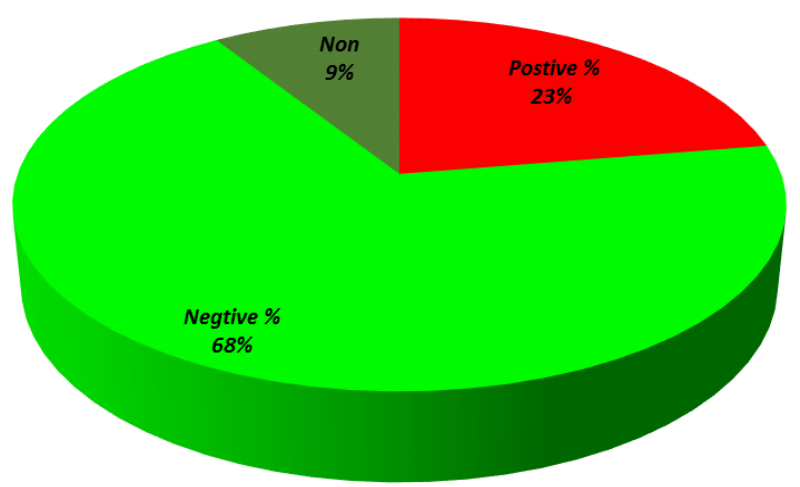

Figure 1. Shows patient frequency in bone scan study.

\section{CT}

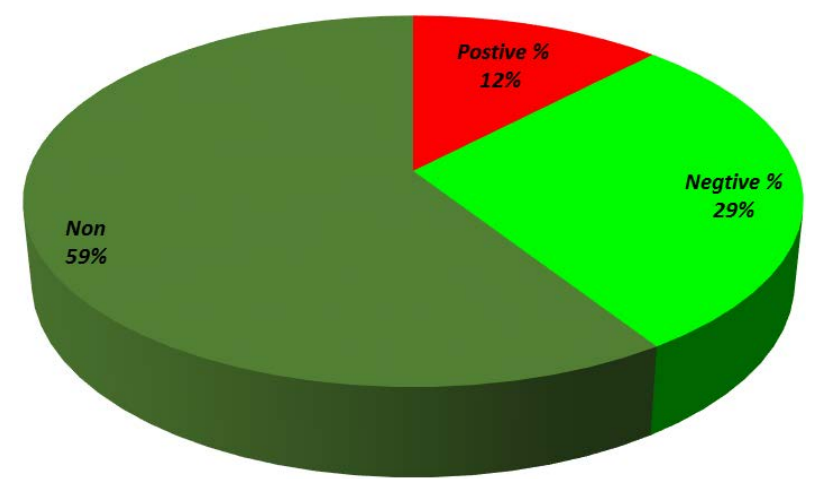

Figure 2. Shows patient frequency in CT scan study. 


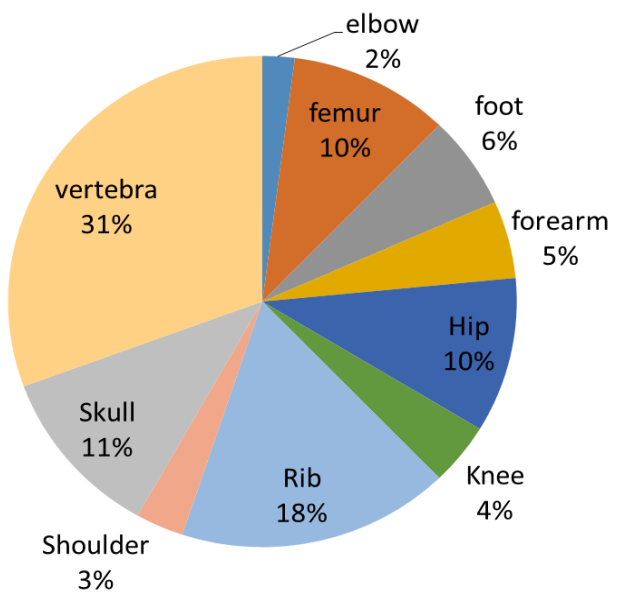

Figure 3. Shows site of metastases in both bone \& CT scan study.

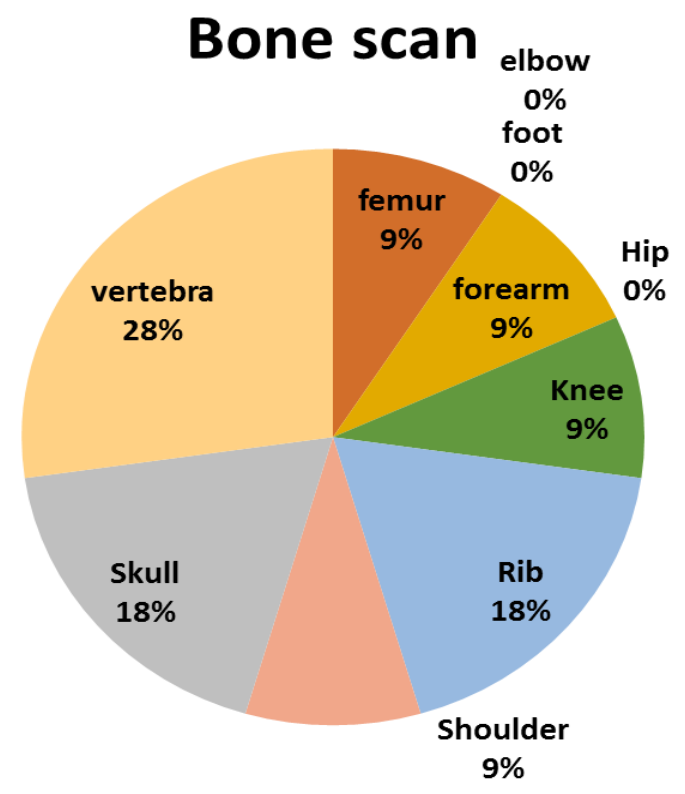

Figure 4. Shows portative finding in bone scan study.

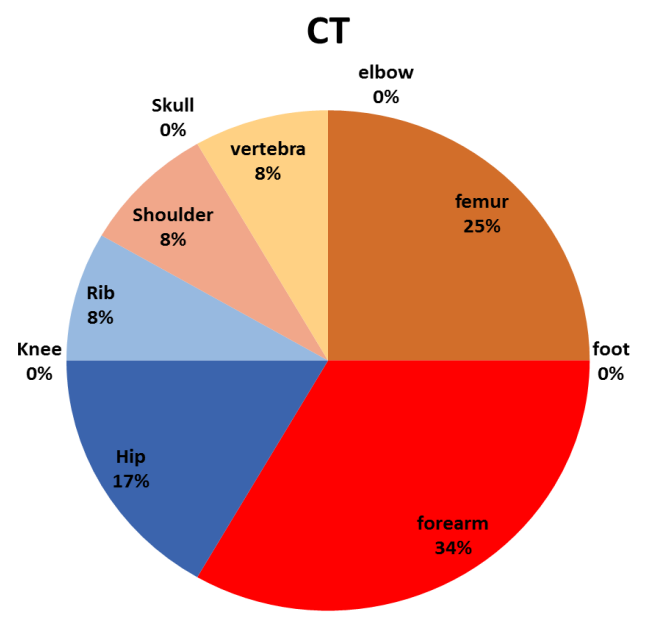

Figure 5. Shows portative finding in CT scan study. 


\section{Sensitivity}

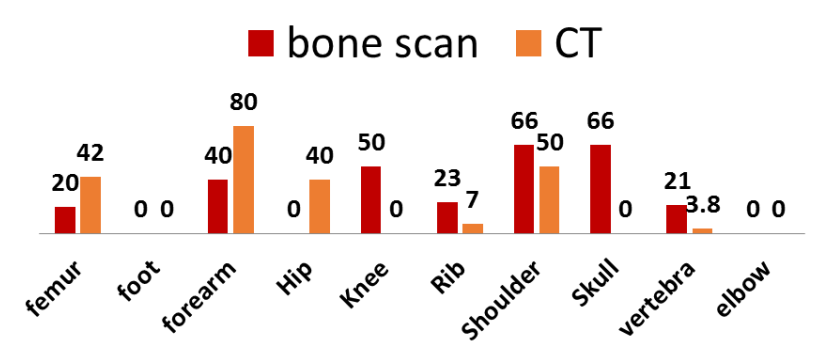

Figure 6. Shows sensitivity of study for both bone \& CT scan.

\section{Bone Scan VS CT Scan Sensitivity}

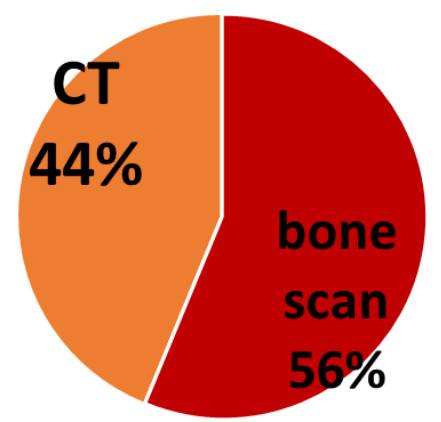

Figure 7. Shows overall sensitivity for both bone \& CT scan.

\section{Discussion}

Dr. Mark S. Pearce et al., state that, CT scans deliver very non-uniform radiation doses across the body. Therefore, assessed the risk of leukaemia and brain tumours in relation to estimated radiation absorbed doses in the appropriate organ (red bone marrow or brain), which were estimated for each type of scan without knowledge of case status. The absorbed dose from a CT scan depends on factors including age, sex, examination type, and year of scan. The retrospective cohort study, show significant associations between the estimated radiation doses provided by CT scans to red bone marrow and brain and subsequent incidence of leukaemia and brain tumours. Assuming typical doses for scans done after 2001 in children aged younger than 15 years, cumulative ionising radiation doses from 2 - 3 head CTs (i.e., $60 \mathrm{mGy}$ ) could almost triple the risk of brain tumours and 5 - 10 head CTs (50 mGy) could triple the risk of leukaemia

Schillaci et al., state that SPECT/CT had a significant impact on the results in scintigraphic imaging interpretation.

\section{Conclusion and Recommendation}

Through the data collection of this research we found that nuclear medicine was more sensitive in detecting bone metastasis (56\%) than CT scan (44\%), and patients are kindly recommended for bone scan study rather than CT scan to minimize radiation hazards.

\section{References}

[1] Haywood, K. (2009) Skeletal System. Marshall Cavendish, New York, 5, 11-12, 17.

[2] Ballard, C. (2009) Bones. Heinemann-Raintree, County Durham, 8, 13. 
[3] Contributors, W. (2006) Human Physiology. Blacksleet River, Philadelphia, 9.

[4] Patel, P.R. (2010) Lecture Notes Radiology. Blackwell, Chichester, 203, 205, 207, 209, 211, 213, 215, 223, 225, 229, 299.

[5] Elgazzar, A.H. (2004) Orthopedic Nuclear Medicine. Springer, Velag Berlin Heidelberg CmbH, 1, 16. http://dx.doi.org/10.1007/978-3-642-18790-2_1

[6] Hsieh, J. (2003) Computed Tomography: Principles, Design, Artifacts, and Recent Advances. Spie, Bellingham, Wash., 26. 
Scientific Research Publishing (SCIRP) is one of the largest Open Access journal publishers. It is currently publishing more than 200 open access, online, peer-reviewed journals covering a wide range of academic disciplines. SCIRP serves the worldwide academic communities and contributes to the progress and application of science with its publication.

Other selected journals from SCIRP are listed as below. Submit your manuscript to us via either submit@scirp.org or Online Submission Portal.
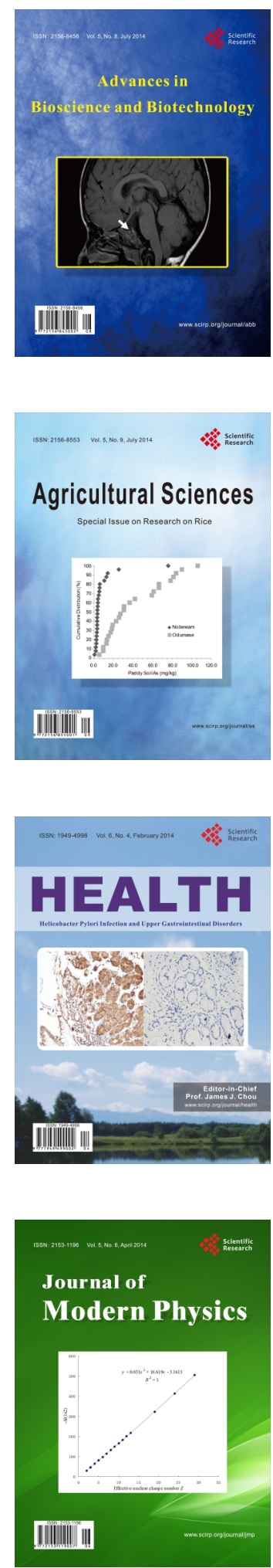
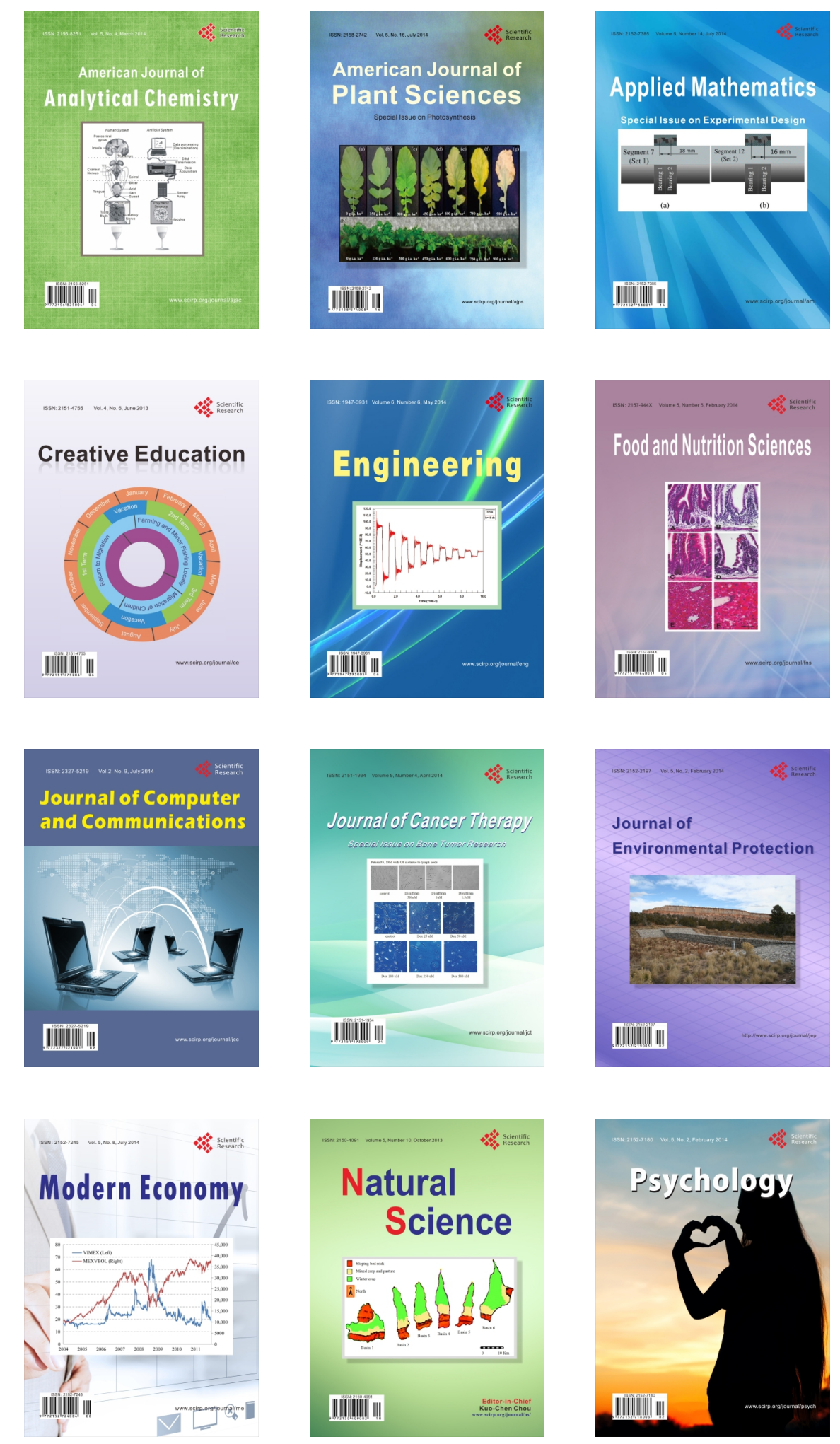